\title{
Wear-Fatigue Test Methods and Their Significance
}

\author{
A. V. Bahdanovich, ${ }^{\text {S S. A. Tyurin, }{ }^{\text {b V. A. Andriyashin, }}{ }^{\text {b }} \text { and A. M. Elavyi }}{ }^{\text {b }}$ \\ a Yanka Kupala State University of Grodno, Grodno, Belarus \\ $\mathrm{b}$ The Joint Institute of Mechanical Engineering, National Academy of Sciences of \\ Belarus, Minsk, Belarus
}

The unified methods of wear-fatigue tests of models of active systems, which are based on a combination of the known mechanical fatigue, friction and wear test methods, are offered. A bending fatigue test method for a unform cylindrical specimen with a test portion diameter of $10 \mathrm{~mm}$ is adopted as a basic one.

Keywords: fatigue, friction, wear-fatigue tests, specimen, counterspecimen.

Introduction. Special wear-fatigue test methods have been elaborated for experimental assessment of mutual and joint influence of friction and fatigue processes on the working capacity of materials and models of active systems under complex loading conditions [1-5].

Under laboratory conditions the wear-fatigue damage resistance is usually studied by testing small models of active systems. The tests are performed on special wear-fatigue test machines of a SI series [5-6].

One of the ways of developing complex wear-fatigue test methods is to combine the known mechanical fatigue test methods with the friction and wear test methods. Figure 1 illustrates, as an example, the principle of such combination whereby a basic fatigue test method incorporates bending with rotation. Note that rotary motion is most typical of modern machines; therefore, the methods as shown in Fig. 1 are of practical importance.

A similar approach enables the machines intended for wear-fatigue tests to be used for conventional tests or mechanical fatigue tests or for friction and wear testing under preset conditions.

The Basic Test Schemes. A test object for mechanical fatigue tests is a structural element, for example, a cylindrical one of a given geometry (Fig. 2c). If the tests are performed in the sliding or rolling friction modes, the test object is a friction pair (Fig. 2b, c) consisting of specimen 1 and counterspecimen 2; they are also called the body and the counterbody. Note that here the specimen is always referred to as the cylindrical structural element and the counterspecimen (counterbody) as the bushing or the roller. Finally, in wear-fatigue tests the test objects are the models of active systems of two elements -1 and 2 (Fig. 2a, d).

It should be mentioned that all the methods of wear-fatigue testing (Fig. 2) implemented on SI series machines are based on using a uniform smooth cylindrical specimen with a test portion diameter $d=2 r=10 \mathrm{~mm}$. It is identical to a standard fatigue test specimen. This provides both the consistency of tests as well as comparability of test results.

Let's address the mechano-sliding fatigue test scheme (Fig. 2e). The cylindrical specimen 1 is fixed in a spindle 2 and rotates with angular speed $\omega_{1}$. 


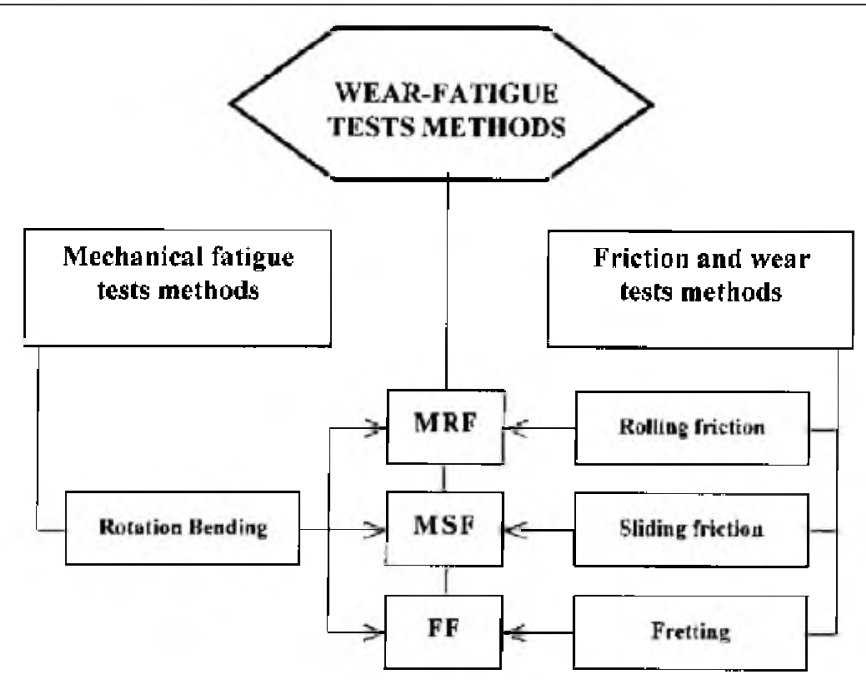

Fig. 1. Development of wear-fatigue test methods: $M R F$ - mechano-rolling fatigue, $M S F$ mechano-sliding fatigue, $F F$ - fretting fatigue.

A vertical bending load $Q$ (upwards or downwards) is applied to its free end. Also, a nonrotating counterspecimen 3 , for example, a plate or a partial bushing, is in contact with the specimen test portion zone of diameter $d=10 \mathrm{Mm}$ under a contact load $F_{N}$. Thus, the maximum contact and bending stresses arise simultaneously in the specimen test portion zone.

Implementation of the test scheme as shown in Fig. 2e makes it possible to perform the following tests:

- wear-fatigue tests for mechano-sliding fatigue (Fig. 2e) with variables $F_{N}$, $Q$, and $\omega_{1}$

- mechanical fatigue tests in bending with rotation (Fig. 2 c) with variables $Q$ and $\omega_{1}$. In this case, the counterspecimen 3 is removed, so $F_{N}=0$;

- sliding friction and wear tests (Fig. 2 c) with variables $F_{N}$ and $\omega$. In this case, no bending loading is applied $(Q=0)$, and specimen 1 is made shorter for the sake of material saving.

In mechanical fatigue tests (Fig. 2c) the bending load $Q$ can be constant (invariable in time $t$ ), but the operating normal stresses at every point of the working section of specimen 1 change during a symmetric cycle (Fig. 3) with period $T$ due to rotation of the specimen.

If the greatest bending moment in the specimen working section is $M=Q l$, where $l$ is the distance from the weakest section to a load action line $Q$; the highest normal stresses in the same section are given by

$$
\sigma=M / W
$$

where $W$ is the moment of resistance.

In sliding friction tests (Fig. 2d), the contact load $F_{N}$ can be static, i.e., constant, but the operating contact stresses are cyclic too. Therefore, these tests are essentially the sliding fatigue tests (under asymmetric tension-compression conditions). 


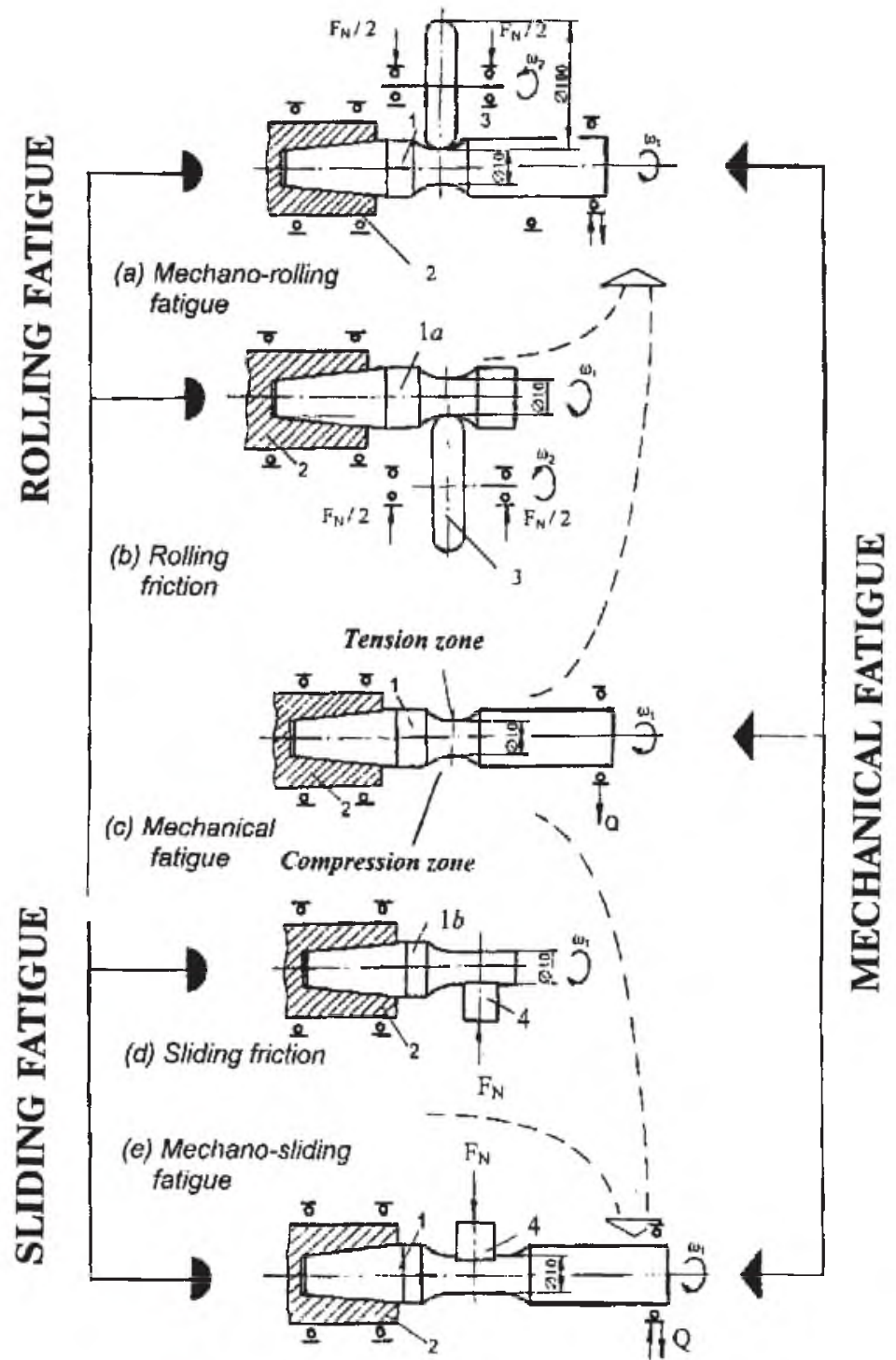

Fig. 2. Typical wear-fatigue test methods: $1,1 a, l b$ - specimens; 2 - test apparatus spindle; $3,4-$ counterspecimena; $Q$ is bending load, $F_{N}$ is contact load, and $\omega_{1}$ and $\omega_{2}$ are rotational speeds of a specimen and counterspecimen, respectively.

The conditions whereby sliding fatigue is realized can be described integrally by either contact loading $F_{N}$ or an average (nominal) contact pressure (2), or a specific sliding friction force called also the frictional stresses (3):

$$
\begin{gathered}
p_{a}=F_{N} / A_{a}, \\
\tau_{W s}=f_{s} p_{a}=F_{s} / A_{a}=f_{s} F_{N} / A_{a},
\end{gathered}
$$

where $A_{a}$ is the nominal area of contact, $F_{s}$ is the sliding friction force, and $f_{s}$ is the coefficient of sliding friction. 
Let us consider the mechano-rolling fatigue test scheme (Fig. 2a). It differs from the mechano-sliding fatigue test scheme (Fig. 2e) in that the fixed bushing is replaced with a rotating roller 3 . Thus, the specimen and the roller can rotate generally with different angular speeds $\omega_{1}$ and $\omega_{2}$ and in different directions.

Realization of the test scheme as shown Fig. 2 a enables one to carry out the following tests:

- wear-fatigue tests for mechano-rolling fatigue (Fig. 2a) with variables $F_{N}$, $Q, \omega_{1}$, and $\omega_{2}$;

- mechanical fatigue tests in bending with rotation (Fig. 2c) with variables $Q$ and $\omega_{1}$. In this case, roller 3 is removed, so $F_{N}=0$ and $\omega_{2}=0$;

- tests in rolling friction or sliding-and-rolling friction (Fig. 2b) with variables $F_{N}, \omega_{1}$, and $\omega_{2}$. In this case, no bending load is applied $(Q=0)$, and specimen 1 is made shorter for the purpose of material saving.

The conditions whereby rolling friction is realized (see Fig. 2b) can be described by either a contact load $F_{N}$, or the highest pressure in the center of a contact area (4) which is defined by the Hertz formula (for a case of elastic deformation), or a specific rolling friction force (5) called also the frictional stress

$$
\begin{gathered}
p_{0}=n_{p} F_{N} / A_{p}, \\
\tau_{W r}=f_{r} p_{0}=F_{r} / A_{a}=f_{r} F_{N} / A_{G},
\end{gathered}
$$

where $A_{p}$ is the area of contact $\left(A_{p}=a^{2}\right.$ for a circular contact area of radius $a$, $A_{p}=l b$ for a band-shaped contact zone measuring $l \times b$, and $A_{p}=a b$ for an elliptic contact area of dimensions $a \times b), n_{p}$ is the factor $\left(n_{p}=0.478\right.$ for circular and elliptic contact areas and $n_{p}=0.637$ for a band-shaped contact zone), $F_{r}$ is the rolling friction force, and $f_{r}$ is the coefficient of rolling friction.

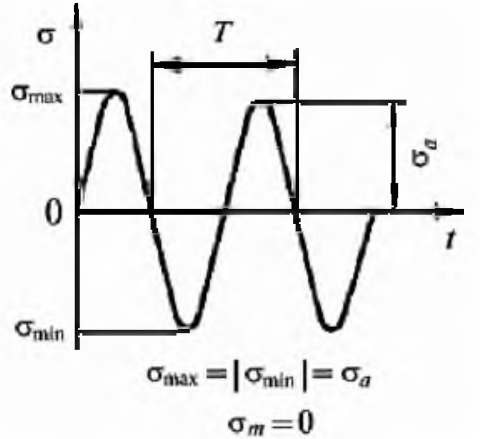

Fig. 3

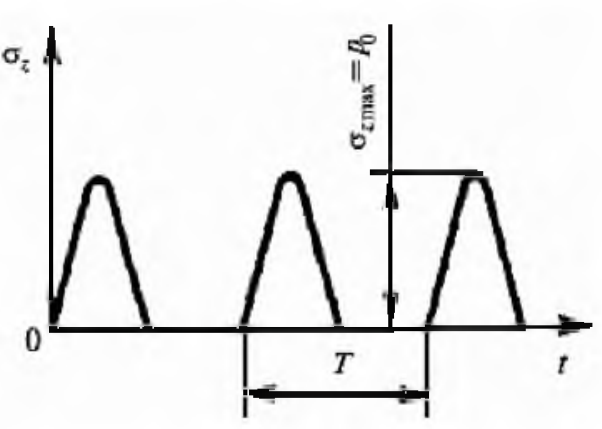

Fig. 4

Fig. 3. A symmetrical stress cycle in mechanical fatigue tests. Fig. 4. Cycle of stresses in rolling fatigue tests.

In rolling friction tests (see Fig. 2b) the contact load $F_{N}$, as in sliding friction, can be static, i.e., constant in time, but the operating contact pressure (for example, $p_{0}=\sigma_{z \max }$ ) is cyclic (Fig. 4). Thus, the rolling friction tests by the scheme in Fig. 2, are essentially the rolling fatigue tests of material surface layer. 
The fretting fatigue test scheme is shown in Fig. 5a. In this case, two counterspecimens 3 called the fretting bridges are pressed with a contact load $F_{N}$ to a test portion of the rotating cylindrical specimen 1 subjected to a bending load $Q$. It can be given circumferential (with a speed $v_{1}$ ) or axial (with a speed $v_{2}$ ) oscillatory movement of small amplitude or to raise both simultaneously to the last.

a

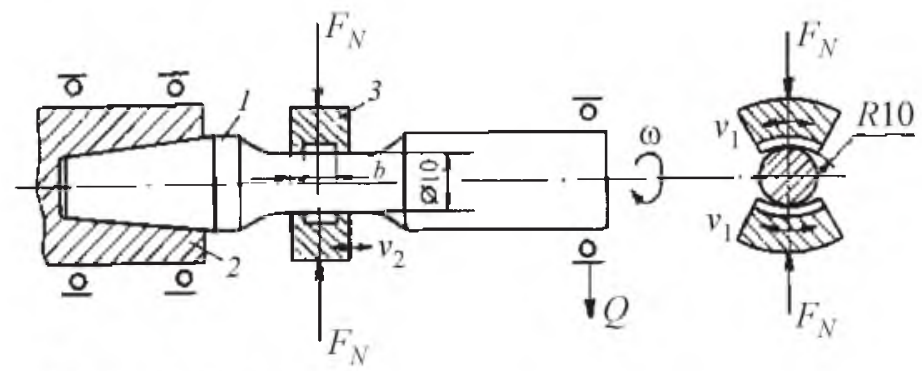

b
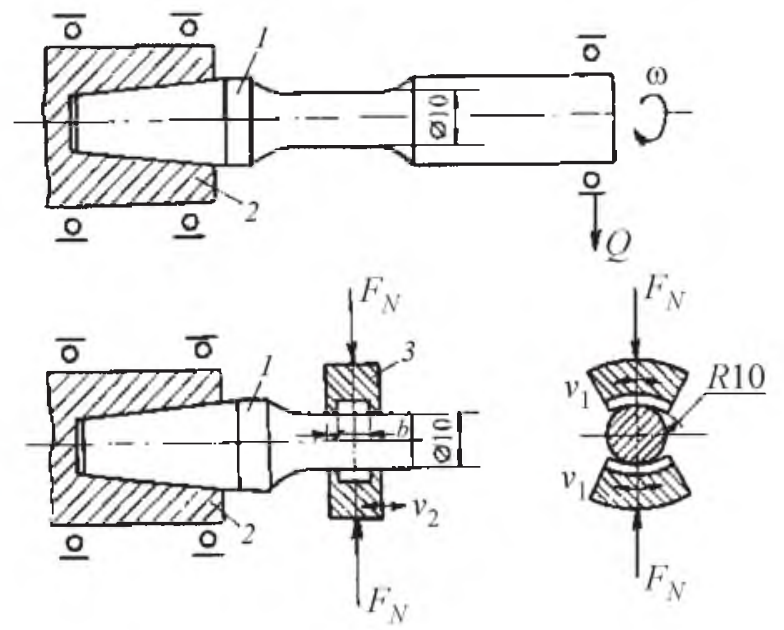

Fig. 5. Test schemes for fretting fatigue (a), mechanical fatigue (b), and fretting (c).

Implementation of the test scheme as shown in Fig. 5 permits the following types of tests:

- wear-fatigue tests for fretting fatigue (see Fig. 5a) with variables $F_{N}, Q$, $\omega, v_{1}$, and $v_{2}$;

- mechanical bending fatigue tests with rotation (see Fig. $5 b$ ) with variables $Q$ and $\omega$. In this case, no fretting bridges are used, so $F_{N}=0, v_{1}=v_{2}=0$;

- fretting tests with axial and/or circumferential sliding (see Fig. $5 \mathrm{c}$ ) with variables $F_{N}, v_{1}$, and $v_{2}$. In this case, no bending load is applied $(Q=0)$, and specimen 1 is made shorter for the purpose of material saving.

The conditions of force interaction between the specimen and the counterspecimen in fretting fatigue can be represented by cyclic stresses (1), frictional stresses (3) or nominal contact pressure,

$$
q=F_{N} / A_{0}
$$

where $A_{0}$ is the initial (nominal) area of contact. 
The Basic Characteristics of Resistance to Wear-Fatigue Damages. The basic characteristics of resistance to wear-fatigue damages are determined by wear-fatigue testing of appropriate objects.

The basic quantitative characteristics of fracture strength are assessed by test results and by plotting a corresponding fatigue curve.

By way of example, Fig. 6 shows four experimental fatigue curves: a mechanical fatigue curve $N\left(\sigma_{a}\right)$ plotted by test results for a specimen of $0.45 \%$ carbon steel (normalized); a rolling fatigue curve $N\left(p_{0}\right)$ constructed by the rolling friction tests results for the pair of 0.45 carbon steel specimen $/ 25 \mathrm{KhGT}$ steel roller (after improvement), and two mechano-rolling fatigue curves plotted by wear-fatigue test results for the active system of $0.45 \%$ carbon steel $/ 25 \mathrm{KhGT}$ steel.

Steel 45 specimen Curve of mechanical fatigue $N\left(\sigma_{a}\right)$
Steel 45 /steel $25 \mathrm{KhGT}$ friction pair. Curve of rolling fatigue $N\left(p_{0}\right)$
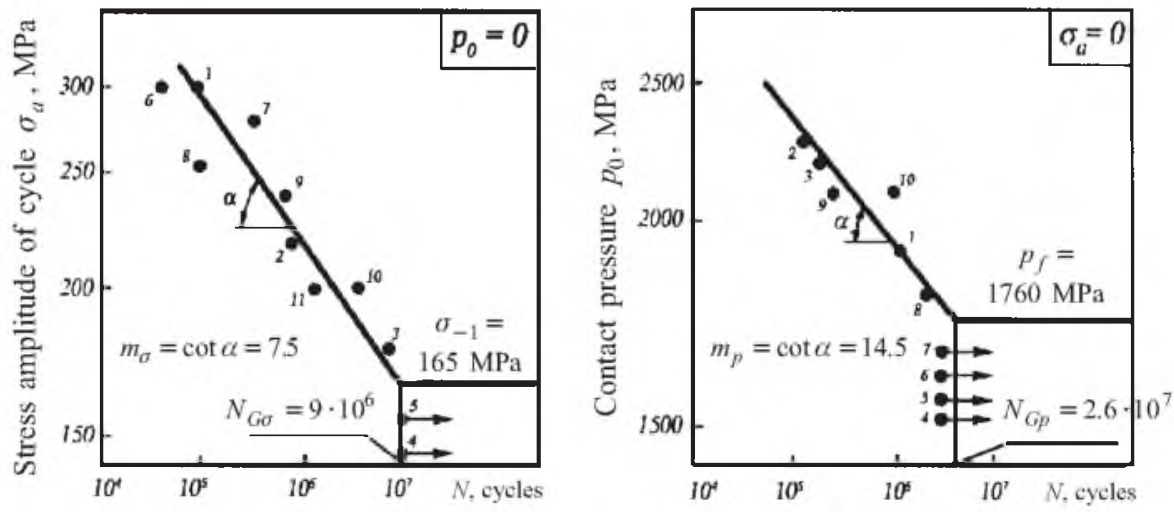

Mechano-rolling fatigue curves

Direct effect $N\left(\sigma_{a}, p_{0}=\mathrm{const}\right)$ Inverse effect $N\left(p_{0}, \sigma_{a}=\right.$ const $)$
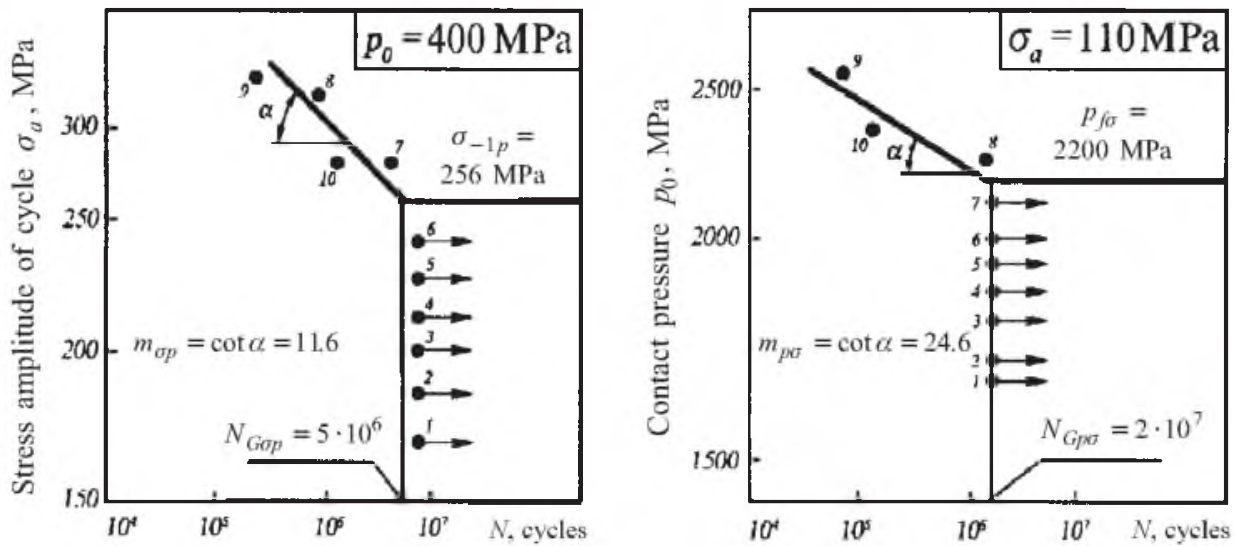

Fig. 6. For determination of basic characteristics of wear-fatigue damages (the point number indicates the sequence of tests).

In the mechanical fatigue tests, disintegration of a specimen serves as a limit state criterion. In rolling fatigue tests, a critical density of pittings on a specimen's test surface is taken as a limit state criterion. The limit states based on damage 
and fracture criteria for mechanical and rolling fatigue tests take place in tests for mechano-rolling fatigue.

In all of the four cases, the fatigue limits $\left(\sigma_{-1}, p_{f}, \sigma_{-1 p}, p_{f \sigma}\right)$, parameters of slope of the left-hand branch of fatigue curves $\left(m_{\sigma}, m_{p}, m_{\sigma p}, m_{p \sigma}\right)$, and the abscissas of critical points of fatigue curves $\left(N_{G \sigma}, N_{G p}, N_{G \sigma p}, N_{G p \sigma}\right)$ are determined. Note that the fatigue limits at mechanical $\left(\sigma_{-1}\right)$ and rolling fatigue $\left(p_{f}\right)$ are unequivocal and unique characteristics of the test objects, while those in mechano-rolling fatigue tests $\left(\sigma_{-1 p}, p_{f \sigma}\right)$ are not. Similar fatigue curves to be plotted can be as many as the number of preset values of parameters $p_{0}=$ const or $\sigma_{a}=$ const in wear-fatigue tests when the mechanisms of direct and back effects are studied.

The influence of friction and wear processes on the variation of mechanical fatigue resistance characteristics can be represented by the direct effect

$$
K_{D}=\sigma_{-1 p} / \sigma_{-1}
$$

In this case, the $K_{D}$ index is a characteristic of strength. For the conditions for which the results are given in Fig. 6 we have $K_{D}=256 / 165=1.62$.

The influence of mechanical fatigue processes on the variation of characteristics of a friction and wear process can be represented by the back effect index

$$
K_{B}=p_{f \sigma} / p_{f}
$$

In this case, the $K_{B}$ index is a tribological characteristic. For the conditions for which the test results are presented in Fig. 6 we have $K_{B}=2200 / 1760=1.25$.

Table 1 provides notations and summarizes numerical values of all the parameters determined by fatigue curves as shown in Fig. 6. A study of these experimental data enables us to make the following conclusions:

(i) the limit stresses in mechano-rolling fatigue are essentially higher than those in mechanical and rolling fatigue $\left(K_{D}>1, K_{B}>1\right)$;

(ii) the fatigue curve exponent increases in passing from the mechanical fatigue curve to the corresponding mechano-rolling fatigue curve $\left(m_{\sigma p}>>m_{\sigma}\right)$ and from the rolling fatigue curve to the corresponding mechano-rolling fatigue curve $\left(m_{p \sigma}>>m_{p}\right)$.

$\mathrm{T}$ a b 1 e 1

System of Notation and Numerical Values of Basic Characteristics

\begin{tabular}{|l|c|c|c|c|}
\hline \multicolumn{1}{|c|}{ Characteristics } & $\begin{array}{c}\text { Mechanical } \\
\text { fatigue curves }\end{array}$ & $\begin{array}{c}\text { Rolling } \\
\text { fatigue curves }\end{array}$ & \multicolumn{2}{|c|}{ Mechano-rolling fatigue curves } \\
\cline { 2 - 5 } & $N\left(\sigma_{a}\right)$ & $N\left(p_{0}\right)$ & $N\left(\sigma_{a}, p_{0}=\right.$ const $)$ & $N\left(p_{0}, \sigma_{a}=\right.$ const $)$ \\
\hline Fatigue limit, MPa & $\sigma_{-1}=165$ & $p_{f}=1760$ & $\sigma_{-1 p}=256$ & $p_{f \sigma}=2200$ \\
\hline $\begin{array}{l}\text { Abscissas of critical } \\
\text { points of fatigue curves, } \\
\text { cycles }\end{array}$ & $N_{G \sigma}=9 \cdot 10^{6}$ & $N_{G p}=2.6 \cdot 10^{7}$ & $N_{G \sigma p}=5 \cdot 10^{6}$ & $N_{G p \sigma}=2 \cdot 10^{7}$ \\
\hline Fatigue curve exponent & $m_{\sigma}=7.5$ & $m_{p}=14.5$ & $m_{\sigma p}=11.6$ & $m_{p \sigma}=24.6$ \\
\hline
\end{tabular}


Differently, under the given experimental conditions the wear-fatigue resistance to damage has turned out to be higher than the mechanical or rolling fatigue resistance.

\section{Conclusions}

1. Unified methods for complex wear-fatigue testing of models of active systems have been developed, which can be implemented using modern machines of a SI series and ensure assessment of fracture strength under preset conditions.

2. New characteristics of resistance to wear-fatigue damages, which are determined from mechano-sliding, mechano-rolling fatigue, and fretting fatigue tests, are proposed.

1. L. A. Sosnovskii, "The method of wear fatigue tests of power systems and their models," Int. J. Frict. Wear, 14, No. 5, 937-952 (1993).

2. K. V. Frolov and N. A. Makhutov, "New test machines and methods," Zavod. Lab., No. 5, 32-33 (1995).

3. M. S. Vysotskii, N. A Makhutov, V. N. Koreshkov, et al., "On development of standard methods for wear-fatigue tests," Ibid, 35-38 (1995).

4. N. A. Mahutov, A. V. Bogdanovich, P. V. Andronov, et al., "Methods of wear-fatigue tests and their realisation on the SI machines," Ibid, No. 6 , $17-42$ (1995).

5. "SI series machines for wear-fatigue tests," in: L. A. Sosnovskii and M. S. Vysotskii (Eds.), Tribo-Fatigue-95: Annual [in Russian], Tribo-Fatigue Ltd., Gomel (1996).

Received 11.06. 2008 